Supporting Information for:

\title{
Characterizing the changes in material use due to vehicle electrification
}

Karan Bhuwalka ${ }^{\dagger}$, Frank R Field III ${ }^{\dagger}$, Robert D. De Kleine ${ }^{\ddagger}$, Hyung Chul Kim ${ }^{\ddagger}$, Timothy J. Wallington ${ }^{\ddagger}$, and Randolph E Kirchain ${ }^{\dagger *}$

${ }^{\dagger}$ Materials Systems Laboratory, Massachusetts Institute of Technology, Cambridge, Massachusetts 02139, United States

* Research and Innovation Center, Ford Motor Company, Dearborn, Michigan 48121, United States

\footnotetext{
* Corresponding Author
}

Telephone: (617) 253-4258

Email: kirchain@mit.edu (R Kirchain) 


\section{SI1. Dataset characteristics}

Table SI.1: Summary of data by source for each vehicle. Percentage defined on total mass after removing duplicates. The large duplicates in the SUV ICEV were due to repeated engine block entries

\begin{tabular}{|c|c|c|c|c|c|c|c|c|}
\hline & Unit & $\begin{array}{l}\text { Sedan } \\
\text { ICEV } 1\end{array}$ & $\begin{array}{l}\text { Sedan } \\
\text { ICEV } 2\end{array}$ & $\begin{array}{l}\text { Sedan } \\
\text { Hybrid }\end{array}$ & $\begin{array}{l}\text { Sedan } \\
\text { PHEV }\end{array}$ & $\begin{array}{l}\text { SUV } \\
\text { ICEV }\end{array}$ & $\begin{array}{l}\text { SUV } \\
\text { Hybrid }\end{array}$ & $\begin{array}{l}\text { SUV } \\
\text { PHEV }\end{array}$ \\
\hline Unreported Part & (\% parts) & $11.6 \%$ & $4.8 \%$ & $2.1 \%$ & $1.6 \%$ & $6.5 \%$ & $4.8 \%$ & $4.5 \%$ \\
\hline Duplicate & (\% mass) & $.5 \%$ & $22 \%$ & $22 \%$ & $7 \%$ & $110 \%$ & $.5 \%$ & $.4 \%$ \\
\hline NoType & (\% mass) & $.71 \%$ & $.67 \%$ & $.69 \%$ & $.73 \%$ & $.29 \%$ & $.58 \%$ & $.72 \%$ \\
\hline ObscureType & (\% mass) & $10.4 \%$ & $10.7 \%$ & $9.9 \%$ & $10.2 \%$ & $4.4 \%$ & $10.2 \%$ & $10.3 \%$ \\
\hline NoFormula & (\% mass) & $5.7 \%$ & $5.8 \%$ & $5.4 \%$ & $4.9 \%$ & $1.4 \%$ & $3.4 \%$ & $3.5 \%$ \\
\hline Original Data & ( $\%$ mass) & $83.19 \%$ & $82.83 \%$ & $84.01 \%$ & $84.17 \%$ & $93.91 \%$ & $85.82 \%$ & $85.48 \%$ \\
\hline
\end{tabular}

Table SI2: Material composition of vehicles in our dataset (after filling in missing data) in grams. Elemental masses reported exclude the weights of the element present in compounds that are reported separately (C in leather)

\begin{tabular}{|c|c|c|c|c|c|c|c|}
\hline Element & $\begin{array}{l}\text { Sedan } \\
\text { ICEV1 }\end{array}$ & $\begin{array}{l}\text { Sedan } \\
\text { ICEV2 }\end{array}$ & $\begin{array}{l}\text { Sedan } \\
\text { Hybrid }\end{array}$ & $\begin{array}{l}\text { Sedan } \\
\text { PHEV }\end{array}$ & $\begin{array}{l}\text { SUV } \\
\text { ICEV }\end{array}$ & $\begin{array}{r}\text { SUV } \\
\text { Hybrid }\end{array}$ & $\begin{array}{r}\text { SUV } \\
\text { PHEV }\end{array}$ \\
\hline $\mathrm{Ag}$ & 37.95 & 18.78 & 30.43 & 35.83 & 70.90 & 97.11 & 75.61 \\
\hline Al & $225,576.06$ & $190,152.44$ & $149,520.83$ & $173,248.74$ & $317,739.65$ & $414,371.46$ & $268,221.37$ \\
\hline $\mathrm{Ar}$ & 174.32 & 168.99 & 168.85 & 197.69 & 58.16 & 60.76 & 29.80 \\
\hline As & 0.19 & 0.17 & 0.32 & 0.25 & 0.24 & 0.51 & 0.59 \\
\hline $\mathrm{Au}$ & 2.29 & 2.87 & 5.25 & 4.65 & 3.37 & 5.41 & 4.18 \\
\hline B & 32.03 & 31.42 & 69.53 & 163.95 & 42.78 & 75.65 & 66.72 \\
\hline $\mathrm{Ba}$ & 290.31 & 315.56 & $1,007.79$ & $1,180.87$ & 588.62 & 576.05 & 427.82 \\
\hline $\mathrm{Be}$ & 0.20 & 0.07 & 40.33 & 39.95 & 0.96 & 0.98 & 1.18 \\
\hline $\mathrm{Bi}$ & 45.17 & 23.43 & 47.72 & 27.40 & 111.32 & 106.89 & 19.57 \\
\hline $\mathrm{Br}$ & 687.10 & 277.98 & 198.33 & 259.23 & 446.02 & 556.12 & 623.98 \\
\hline C (Organic) & $188,476.91$ & $162,988.89$ & $168,423.70$ & $197,630.57$ & $248,781.04$ & $269,940.93$ & $283,717.79$ \\
\hline $\mathrm{Ca}$ & $4,529.47$ & $3,594.20$ & $4,717.15$ & $4,718.43$ & $6,019.85$ & $5,991.39$ & $4,500.13$ \\
\hline Cd & 0.36 & 0.37 & 0.91 & 1.14 & 0.35 & 0.90 & 0.41 \\
\hline $\mathrm{Ce}$ & 5.15 & 35.13 & 5.05 & 5.17 & 3.11 & 781.96 & 85.63 \\
\hline $\mathrm{Cl}$ & $5,760.35$ & $3,963.18$ & $6,617.34$ & $6,246.30$ & $9,151.50$ & $7,399.24$ & $8,344.05$ \\
\hline Co & 77.74 & 57.85 & 689.50 & $3,529.21$ & 124.56 & 985.59 & $8,011.93$ \\
\hline $\mathrm{Cr}$ & $10,004.92$ & $6,543.76$ & $7,164.83$ & $6,695.89$ & $10,591.95$ & $10,911.17$ & $12,579.96$ \\
\hline Cs & 3.97 & 4.26 & 4.62 & 4.26 & 0.04 & 0.06 & 0.04 \\
\hline $\mathrm{Cu}$ & $30,922.43$ & $30,484.51$ & $52,815.72$ & $53,913.68$ & $42,817.29$ & $67,375.51$ & $75,520.07$ \\
\hline D & - & - & 0.00 & 0.00 & 0.00 & 0.00 & - \\
\hline Dy & 4.47 & 4.45 & 10.89 & 10.21 & 7.57 & 76.66 & 61.49 \\
\hline Eu & - & 0.00 & - & 0.00 & - & - & - \\
\hline $\mathbf{F}$ & $5,895.22$ & $12,237.32$ & $5,301.74$ & 446.15 & $8,051.50$ & $5,942.00$ & $6,967.91$ \\
\hline $\mathrm{Fe}$ & $1,051,158.79$ & $1,009,971.09$ & $1,108,479.12$ & $1,092,316.66$ & $\begin{array}{r}1,203,356.1 \\
6\end{array}$ & $\begin{array}{r}1,468,610.9 \\
3\end{array}$ & $\begin{array}{r}1,497,233.7 \\
6\end{array}$ \\
\hline Ga & 0.35 & 0.35 & 3.20 & 0.35 & 0.71 & 3.07 & 3.03 \\
\hline Gd & 0.00 & 0.00 & 0.02 & 0.00 & 0.00 & 0.00 & 0.00 \\
\hline Ge & 0.01 & 0.00 & 0.00 & 0.01 & 0.02 & 0.02 & 0.03 \\
\hline Graphite & 74.86 & 89.04 & $1,857.62$ & $9,085.23$ & 79.40 & $2,013.98$ & $13,395.28$ \\
\hline H & $23,626.56$ & $19,150.09$ & $20,683.87$ & $25,841.09$ & $31,458.32$ & $34,144.10$ & $36,792.34$ \\
\hline $\mathrm{He}$ & 5.61 & 5.44 & 5.44 & 8.36 & 2.13 & 2.21 & 1.19 \\
\hline Hf & 0.03 & 0.02 & 0.00 & 0.00 & 0.00 & 0.00 & 0.02 \\
\hline $\mathrm{Hg}$ & 0.11 & 0.17 & 0.12 & 0.11 & 0.04 & 0.04 & 0.04 \\
\hline Ho & - & - & - & - & - & 0.07 & 0.07 \\
\hline 1 & 1.58 & 1.69 & 1.45 & 2.59 & 0.76 & 0.61 & 0.63 \\
\hline In & 0.58 & 0.58 & 0.59 & 0.62 & 1.41 & 1.42 & 1.14 \\
\hline Ir & 0.00 & 0.01 & 0.02 & 0.02 & 0.02 & 0.00 & 0.03 \\
\hline K & 70.51 & 140.22 & 91.71 & 77.59 & 58.93 & 104.14 & 45.22 \\
\hline $\mathrm{Kr}$ & 0.40 & 0.51 & 0.59 & 0.00 & 0.00 & - & 0.00 \\
\hline La & 2.79 & 15.19 & 2.88 & 0.14 & 21.27 & 36.03 & 14.92 \\
\hline Leather & - & 1.58 & 0.00 & $1,176.76$ & $1,932.88$ & $1,932.94$ & $1,564.81$ \\
\hline
\end{tabular}




\begin{tabular}{|c|c|c|c|c|c|c|c|}
\hline Li & 11.75 & 11.54 & 252.26 & $1,336.79$ & 15.74 & 104.05 & 929.62 \\
\hline Lu & 0.00 & 0.00 & 0.00 & 0.00 & 0.00 & - & 0.00 \\
\hline Mg & $5,080.28$ & $5,015.97$ & $4,327.20$ & $5,229.06$ & $17,841.24$ & $18,718.97$ & $17,666.42$ \\
\hline $\mathrm{Mn}$ & $7,229.73$ & $7,437.00$ & $7,785.38$ & $10,193.60$ & $10,944.33$ & $12,970.75$ & $19,506.38$ \\
\hline Mo & 310.44 & 304.01 & 326.48 & 411.83 & 608.52 & 792.55 & 754.48 \\
\hline $\mathbf{N}$ & $4,619.70$ & $3,926.77$ & $5,346.28$ & $5,485.53$ & $12,809.42$ & $13,311.55$ & $7,116.04$ \\
\hline $\mathrm{Na}$ & $4,897.25$ & $4,355.51$ & $4,651.25$ & $4,201.61$ & $5,032.61$ & $5,262.72$ & $4,040.80$ \\
\hline Natural Rubber & $1,477.46$ & $8,907.69$ & $8,794.79$ & $8,898.15$ & $13,904.56$ & $16,104.41$ & $14,642.22$ \\
\hline Nb & 129.60 & 85.64 & 91.29 & 198.37 & 287.04 & 249.40 & 264.51 \\
\hline Nd & 95.18 & 114.90 & $1,122.36$ & 628.50 & 100.71 & 829.82 & 981.99 \\
\hline $\mathbf{N i}$ & $3,283.41$ & $2,641.05$ & $2,653.48$ & $5,530.96$ & $3,725.00$ & $4,184.45$ & $12,239.11$ \\
\hline 0 & $75,434.35$ & $69,489.54$ & $73,809.16$ & $83,514.23$ & $96,421.21$ & $100,035.28$ & $91,195.32$ \\
\hline $\mathbf{P}$ & 446.21 & 396.38 & 395.21 & 514.59 & 432.72 & 556.84 & 644.41 \\
\hline $\mathrm{Pb}$ & $8,926.35$ & $17,455.91$ & $8,890.09$ & $8,924.25$ & $14,829.67$ & $17,135.22$ & $17,116.21$ \\
\hline Pd & 3.64 & 4.63 & 2.13 & 2.20 & 4.80 & 6.87 & 0.80 \\
\hline $\operatorname{Pr}$ & 5.80 & 6.85 & 5.07 & 6.02 & 17.44 & 183.03 & 254.18 \\
\hline Pt & 0.20 & 0.21 & 0.27 & 0.22 & 0.04 & 0.05 & 0.09 \\
\hline Pure C (steel alloy) & $6,540.99$ & $4,611.50$ & $5,268.86$ & $5,242.09$ & $5,064.92$ & $7,409.97$ & $6,870.89$ \\
\hline $\mathbf{R b}$ & - & - & - & - & 0.00 & 0.00 & 0.00 \\
\hline $\operatorname{Re}$ & - & - & - & - & 0.00 & 0.00 & 0.00 \\
\hline $\mathbf{R h}$ & 0.29 & 0.23 & 0.22 & 0.22 & 0.32 & 0.29 & 0.00 \\
\hline Ru & 0.08 & 0.08 & 0.10 & 0.12 & 0.03 & 0.03 & 0.05 \\
\hline $\mathbf{S}$ & $2,130.15$ & $1,360.14$ & $1,896.08$ & $1,928.93$ & $1,332.78$ & $1,762.04$ & $1,654.65$ \\
\hline Sb & 89.04 & 75.44 & 153.03 & 280.67 & 208.66 & 274.47 & 287.63 \\
\hline $\mathrm{Se}$ & 0.09 & 0.09 & 0.05 & 0.21 & 53.34 & 0.30 & 0.06 \\
\hline Sheet Mica & 419.83 & 395.53 & 429.23 & 389.28 & 381.30 & 406.14 & 363.82 \\
\hline Si & $45,164.15$ & $43,008.07$ & $43,408.38$ & $45,975.05$ & $53,352.01$ & $69,454.57$ & $51,622.68$ \\
\hline Sm & 5.83 & 5.36 & 4.89 & 4.46 & 1.67 & 0.66 & 0.52 \\
\hline Sn & 539.62 & 564.55 & 588.28 & 581.04 & 828.19 & $1,592.99$ & $1,126.80$ \\
\hline $\mathrm{Sr}$ & 251.60 & 345.73 & 252.88 & 545.06 & 407.00 & 516.10 & 511.45 \\
\hline $\mathrm{Ta}$ & 7.95 & 7.67 & 8.44 & 15.59 & 10.82 & 13.01 & 17.45 \\
\hline Tb & 0.00 & 0.00 & 0.86 & 0.87 & 3.17 & 11.28 & 13.43 \\
\hline Te & 0.20 & 0.20 & 0.27 & 5.97 & 53.17 & 0.10 & 0.10 \\
\hline Th & - & - & 0.00 & 0.00 & 0.00 & 0.00 & 0.00 \\
\hline $\mathrm{Ti}$ & 800.82 & 756.47 & 643.71 & 819.71 & $1,570.82$ & $1,158.94$ & 915.09 \\
\hline $\mathrm{TI}$ & 0.00 & 0.00 & 0.00 & 0.00 & 0.00 & 0.00 & 0.00 \\
\hline Tm & 0.00 & 0.00 & 0.00 & 0.00 & 0.22 & 0.35 & 0.35 \\
\hline $\begin{array}{l}\text { Unspecified } \\
\text { Organic }\end{array}$ & $79,744.02$ & $80,565.44$ & $75,536.91$ & $71,108.80$ & $46,596.81$ & $51,973.29$ & $52,581.17$ \\
\hline V & 57.41 & 55.85 & 62.88 & 172.96 & 287.69 & 300.85 & 306.44 \\
\hline $\mathbf{W}$ & 23.06 & 3.28 & 6.62 & 5.12 & 22.44 & 9.24 & 24.76 \\
\hline $\mathrm{Xe}$ & 0.15 & 0.19 & 0.21 & - & 0.00 & - & 0.00 \\
\hline $\mathbf{Y}$ & 0.16 & 4.00 & 0.12 & 0.12 & 0.01 & 13.45 & 0.04 \\
\hline $\mathrm{Yb}$ & - & - & 0.02 & 0.02 & - & 0.03 & 0.03 \\
\hline $\mathrm{Zn}$ & $19,923.28$ & $20,036.82$ & $24,299.32$ & $24,205.65$ & $13,428.47$ & $14,868.65$ & $15,029.38$ \\
\hline $\mathrm{Zr}$ & 159.87 & 207.90 & 167.58 & 135.67 & 263.79 & 310.47 & 173.23 \\
\hline Total & $1,815,278.7$ & $1,712,439.7$ & $1,799,149.1$ & $1,863,362.6$ & $2,182,365.5$ & $2,632,629.1$ & $2,537,165.3$ \\
\hline
\end{tabular}


Table SI.3: Weight of each material (in grams) in an average vehicle from a PHEV fleet (SUVs and Sedans in a ratio of 2:1). Data disaggregated by proportion from different sources after filling in algorithms

\begin{tabular}{|c|c|c|c|c|c|}
\hline Material & Weight (g) & ObscureType & NoType & $\begin{array}{r}\text { Original } \\
\text { Data }\end{array}$ & Unreported \\
\hline $\mathrm{Ag}$ & 62.48 & $0.0 \%$ & $4.8 \%$ & $67.7 \%$ & $27.5 \%$ \\
\hline Al & 236880.45 & $0.0 \%$ & $0.8 \%$ & $83.1 \%$ & $16.1 \%$ \\
\hline $\mathrm{Ar}$ & 85.21 & $0.0 \%$ & $2.3 \%$ & $97.7 \%$ & $0.0 \%$ \\
\hline As & 0.48 & $0.0 \%$ & $3.0 \%$ & $95.7 \%$ & $1.4 \%$ \\
\hline $\mathrm{Au}$ & 4.33 & $0.0 \%$ & $10.9 \%$ & $75.7 \%$ & $13.4 \%$ \\
\hline B & 98.81 & $0.0 \%$ & $1.7 \%$ & $97.5 \%$ & $0.9 \%$ \\
\hline $\mathrm{Ba}$ & 676.32 & $0.0 \%$ & $3.3 \%$ & $95.9 \%$ & $0.8 \%$ \\
\hline $\mathrm{Be}$ & 13.97 & $0.0 \%$ & $6.4 \%$ & $93.6 \%$ & $0.0 \%$ \\
\hline $\mathrm{Bi}$ & 22.15 & $0.0 \%$ & $3.6 \%$ & $91.6 \%$ & $4.8 \%$ \\
\hline $\mathrm{Br}$ & 503.61 & $42.5 \%$ & $5.6 \%$ & $49.8 \%$ & $2.1 \%$ \\
\hline C (Organics) & 233309.01 & $44.9 \%$ & $3.7 \%$ & $50.3 \%$ & $1.1 \%$ \\
\hline $\mathrm{Ca}$ & 4572.17 & $0.0 \%$ & $5.1 \%$ & $94.6 \%$ & $0.3 \%$ \\
\hline Cd & 0.66 & $0.0 \%$ & $2.7 \%$ & $94.2 \%$ & $3.2 \%$ \\
\hline $\mathrm{Ce}$ & 59.08 & $0.0 \%$ & $0.9 \%$ & $99.1 \%$ & $0.0 \%$ \\
\hline $\mathrm{Cl}$ & 7651.79 & $55.3 \%$ & $5.7 \%$ & $38.3 \%$ & $0.6 \%$ \\
\hline Co & 6532.63 & $0.0 \%$ & $2.6 \%$ & $97.2 \%$ & $0.1 \%$ \\
\hline $\mathrm{Cr}$ & 10638.22 & $5.4 \%$ & $1.3 \%$ & $90.8 \%$ & $2.5 \%$ \\
\hline Cs & 1.43 & $0.0 \%$ & $1.2 \%$ & $98.8 \%$ & $0.0 \%$ \\
\hline $\mathrm{Cu}$ & 68389.96 & $0.0 \%$ & $3.2 \%$ & $92.9 \%$ & $3.8 \%$ \\
\hline D & 0.00 & $0.0 \%$ & $100.0 \%$ & $0.0 \%$ & $0.0 \%$ \\
\hline Dy & 44.57 & $0.0 \%$ & $2.6 \%$ & $97.3 \%$ & $0.1 \%$ \\
\hline Eu & 0.00 & $0.0 \%$ & $0.2 \%$ & $99.8 \%$ & $0.0 \%$ \\
\hline $\mathbf{F}$ & 4815.73 & $68.8 \%$ & $3.6 \%$ & $26.9 \%$ & $0.8 \%$ \\
\hline $\mathrm{Fe}$ & 1363611.03 & $0.0 \%$ & $0.5 \%$ & $93.6 \%$ & $5.8 \%$ \\
\hline $\mathrm{Ga}$ & 2.15 & $0.0 \%$ & $3.5 \%$ & $96.5 \%$ & $0.0 \%$ \\
\hline Gd & 0.00 & $0.0 \%$ & $1.4 \%$ & $98.6 \%$ & $0.0 \%$ \\
\hline Ge & 0.02 & $0.0 \%$ & $10.7 \%$ & $89.2 \%$ & $0.1 \%$ \\
\hline Graphite & 11972.96 & $0.0 \%$ & $2.4 \%$ & $97.6 \%$ & $0.0 \%$ \\
\hline H & 33178.43 & $48.5 \%$ & $3.6 \%$ & $47.2 \%$ & $0.7 \%$ \\
\hline $\mathrm{He}$ & 3.55 & $0.0 \%$ & $1.9 \%$ & $98.1 \%$ & $0.0 \%$ \\
\hline Hf & 0.02 & $0.0 \%$ & $6.9 \%$ & $93.1 \%$ & $0.0 \%$ \\
\hline $\mathrm{Hg}$ & 0.06 & $0.0 \%$ & $1.1 \%$ & $60.2 \%$ & $38.7 \%$ \\
\hline Ho & 0.05 & $0.0 \%$ & $0.9 \%$ & $99.1 \%$ & $0.0 \%$ \\
\hline I & 1.28 & $0.0 \%$ & $4.3 \%$ & $94.2 \%$ & $1.4 \%$ \\
\hline In & 0.97 & $0.0 \%$ & $2.9 \%$ & $95.9 \%$ & $1.2 \%$ \\
\hline Ir & 0.03 & $0.0 \%$ & $0.6 \%$ & $99.4 \%$ & $0.0 \%$ \\
\hline K & 55.90 & $0.0 \%$ & $1.5 \%$ & $55.3 \%$ & $43.1 \%$ \\
\hline $\mathrm{Kr}$ & 0.00 & $0.0 \%$ & $74.3 \%$ & $25.7 \%$ & $0.0 \%$ \\
\hline La & 10.04 & $0.0 \%$ & $1.1 \%$ & $98.2 \%$ & $0.7 \%$ \\
\hline Leather & 1436.75 & $0.0 \%$ & $2.6 \%$ & $97.4 \%$ & $0.0 \%$ \\
\hline Li & 1063.99 & $0.0 \%$ & $2.2 \%$ & $97.7 \%$ & $0.1 \%$ \\
\hline Lu & 0.00 & $0.0 \%$ & $0.2 \%$ & $99.8 \%$ & $0.0 \%$ \\
\hline $\mathrm{Mg}$ & 13562.09 & $5.9 \%$ & $2.7 \%$ & $90.3 \%$ & $1.1 \%$ \\
\hline Mn & 16433.17 & $0.0 \%$ & $1.3 \%$ & $95.3 \%$ & $3.4 \%$ \\
\hline Mo & 641.40 & $0.0 \%$ & $0.6 \%$ & $97.2 \%$ & $2.2 \%$ \\
\hline $\mathbf{N}$ & 6577.97 & $51.0 \%$ & $6.1 \%$ & $41.5 \%$ & $1.5 \%$ \\
\hline $\mathrm{Na}$ & 4093.87 & $2.3 \%$ & $3.5 \%$ & $94.1 \%$ & $0.1 \%$ \\
\hline Natural Rubber & 12746.68 & $0.0 \%$ & $15.5 \%$ & $84.5 \%$ & $0.0 \%$ \\
\hline $\mathrm{Nb}$ & 242.69 & $0.0 \%$ & $0.7 \%$ & $94.5 \%$ & $4.8 \%$ \\
\hline Nd & 865.34 & $0.0 \%$ & $1.7 \%$ & $97.6 \%$ & $0.7 \%$ \\
\hline $\mathrm{Ni}$ & 10025.42 & $0.0 \%$ & $2.0 \%$ & $96.5 \%$ & $1.5 \%$ \\
\hline 0 & 88660.57 & $33.0 \%$ & $3.3 \%$ & $62.8 \%$ & $0.9 \%$ \\
\hline $\mathbf{P}$ & 601.57 & $0.0 \%$ & $0.6 \%$ & $97.6 \%$ & $1.8 \%$ \\
\hline $\mathrm{Pb}$ & 14412.86 & $0.0 \%$ & $1.3 \%$ & $98.6 \%$ & $0.2 \%$ \\
\hline Pd & 1.26 & $0.0 \%$ & $18.1 \%$ & $80.2 \%$ & $1.7 \%$ \\
\hline $\operatorname{Pr}$ & 172.28 & $0.0 \%$ & $1.9 \%$ & $98.0 \%$ & $0.0 \%$ \\
\hline
\end{tabular}




\begin{tabular}{|c|c|c|c|c|c|}
\hline Pt & 0.13 & $0.0 \%$ & $1.9 \%$ & $98.0 \%$ & $0.1 \%$ \\
\hline Pure C & 6333.39 & $0.0 \%$ & $0.6 \%$ & $99.4 \%$ & $0.0 \%$ \\
\hline Rb & 0.00 & $0.0 \%$ & $1.3 \%$ & $98.7 \%$ & $0.0 \%$ \\
\hline $\mathrm{Re}$ & 0.00 & $0.0 \%$ & $1.9 \%$ & $98.1 \%$ & $0.0 \%$ \\
\hline $\mathbf{R h}$ & 0.08 & $0.0 \%$ & $0.1 \%$ & $99.9 \%$ & $0.0 \%$ \\
\hline Ru & 0.07 & $0.0 \%$ & $4.8 \%$ & $90.8 \%$ & $4.5 \%$ \\
\hline $\mathbf{S}$ & 1745.16 & $1.1 \%$ & $3.3 \%$ & $94.2 \%$ & $1.5 \%$ \\
\hline $\mathrm{Sb}$ & 285.33 & $0.0 \%$ & $6.7 \%$ & $92.8 \%$ & $0.5 \%$ \\
\hline Se & 0.11 & $0.0 \%$ & $5.4 \%$ & $94.4 \%$ & $0.2 \%$ \\
\hline Sheet Mica & 372.22 & $0.0 \%$ & $39.7 \%$ & $60.3 \%$ & $0.0 \%$ \\
\hline Si & 49758.96 & $17.7 \%$ & $1.6 \%$ & $72.1 \%$ & $8.6 \%$ \\
\hline Sm & 1.82 & $0.0 \%$ & $2.3 \%$ & $20.8 \%$ & $76.8 \%$ \\
\hline Sn & 946.70 & $0.0 \%$ & $3.8 \%$ & $88.0 \%$ & $8.3 \%$ \\
\hline $\mathrm{Sr}$ & 522.54 & $0.0 \%$ & $2.1 \%$ & $92.3 \%$ & $5.7 \%$ \\
\hline $\mathrm{Ta}$ & 16.84 & $0.0 \%$ & $7.1 \%$ & $87.1 \%$ & $5.8 \%$ \\
\hline $\mathrm{Tb}$ & 9.28 & $0.0 \%$ & $1.4 \%$ & $98.6 \%$ & $0.0 \%$ \\
\hline Te & 2.04 & $0.0 \%$ & $3.7 \%$ & $96.2 \%$ & $0.0 \%$ \\
\hline Th & 0.00 & $0.0 \%$ & $17.5 \%$ & $82.5 \%$ & $0.0 \%$ \\
\hline $\mathrm{Ti}$ & 883.62 & $2.5 \%$ & $1.9 \%$ & $89.8 \%$ & $5.9 \%$ \\
\hline TI & 0.00 & $0.0 \%$ & $1.7 \%$ & $98.3 \%$ & $0.0 \%$ \\
\hline $\mathrm{Tm}$ & 0.24 & $0.0 \%$ & $0.1 \%$ & $65.2 \%$ & $34.7 \%$ \\
\hline Unspecified Organics & 58695.29 & $0.0 \%$ & $7.1 \%$ & $92.9 \%$ & $0.0 \%$ \\
\hline V & 262.39 & $0.0 \%$ & $0.6 \%$ & $91.8 \%$ & $7.7 \%$ \\
\hline $\mathbf{W}$ & 18.28 & $0.0 \%$ & $0.2 \%$ & $94.7 \%$ & $5.0 \%$ \\
\hline $\mathrm{Xe}$ & 0.00 & $0.0 \%$ & $100.0 \%$ & $0.0 \%$ & $0.0 \%$ \\
\hline $\mathbf{Y}$ & 0.07 & $0.0 \%$ & $11.5 \%$ & $86.0 \%$ & $2.6 \%$ \\
\hline $\mathbf{Y b}$ & 0.03 & $0.0 \%$ & $0.7 \%$ & $99.3 \%$ & $0.0 \%$ \\
\hline Zn & 18057.55 & $0.0 \%$ & $1.2 \%$ & $95.8 \%$ & $2.9 \%$ \\
\hline $\mathrm{Zr}$ & 160.83 & $0.0 \%$ & $1.0 \%$ & $98.6 \%$ & $0.5 \%$ \\
\hline
\end{tabular}




\section{SI 2. Pricing assumptions used}

Table SI.4: Average Prices and Price Volatilities used in the analysis. For all elements, price data taken from USGS between the years 1998-2015 as reported by Theler et al. Line fit after adjusting for inflation to 1998 prices. For non-elemental compounds the prices we used are described below the table.

Element Average Price

\begin{tabular}{|c|c|c|c|c|}
\hline & & & & \\
\hline$A g$ & $3.41 \mathrm{E}+02$ & $3.88 \mathrm{E}-01$ & $3.12 \mathrm{E}+01$ & $6.04 \mathrm{E}+01$ \\
\hline Al & $1.61 \mathrm{E}+00$ & $1.75 \mathrm{E}-01$ & $6.75 \mathrm{E}-03$ & $1.55 \mathrm{E}+00$ \\
\hline As & $5.56 \mathrm{E}-01$ & $2.31 \mathrm{E}-01$ & $-1.96 \mathrm{E}-02$ & 7.32E-01 \\
\hline$A u$ & $1.98 \mathrm{E}+04$ & $2.36 \mathrm{E}-01$ & $1.83 \mathrm{E}+03$ & $3.30 \mathrm{E}+03$ \\
\hline$B$ & $3.14 \mathrm{E}-01$ & $1.98 \mathrm{E}-01$ & $2.60 \mathrm{E}-02$ & $8.00 \mathrm{E}-02$ \\
\hline$B a$ & $4.56 \mathrm{E}-02$ & $2.33 \mathrm{E}-01$ & 4.39E-03 & $6.09 \mathrm{E}-03$ \\
\hline$B e$ & $1.94 \mathrm{E}+02$ & $7.58 \mathrm{E}-02$ & $9.51 \mathrm{E}+00$ & $1.71 \mathrm{E}+02$ \\
\hline$B i$ & $1.21 \mathrm{E}+01$ & $3.93 \mathrm{E}-01$ & $6.33 \mathrm{E}-01$ & $6.38 \mathrm{E}+00$ \\
\hline $\mathrm{Ca}$ & $6.48 \mathrm{E}-03$ & $6.33 \mathrm{E}-02$ & $1.96 \mathrm{E}-04$ & $4.72 \mathrm{E}-03$ \\
\hline$C d$ & $1.88 \mathrm{E}+00$ & $7.82 \mathrm{E}-01$ & $7.88 \mathrm{E}-02$ & $1.17 \mathrm{E}+00$ \\
\hline $\mathrm{Ce}$ & $3.24 \mathrm{E}+01$ & $6.55 \mathrm{E}-01$ & $-7.39 \mathrm{E}-01$ & $3.41 \mathrm{E}+01$ \\
\hline Co & $3.08 \mathrm{E}+01$ & $4.23 \mathrm{E}-01$ & $-3.13 \mathrm{E}-01$ & $3.36 \mathrm{E}+01$ \\
\hline $\mathrm{Cr}$ & $1.16 \mathrm{E}-01$ & 4.07E-01 & $5.73 \mathrm{E}-03$ & $6.46 \mathrm{E}-02$ \\
\hline $\mathrm{Cu}$ & $3.92 \mathrm{E}+00$ & $2.87 \mathrm{E}-01$ & $2.83 \mathrm{E}-01$ & $1.37 \mathrm{E}+00$ \\
\hline$D y$ & $2.87 \mathrm{E}+02$ & $1.01 \mathrm{E}+00$ & $2.80 \mathrm{E}+01$ & $-7.68 \mathrm{E}+00$ \\
\hline$E r$ & $1.34 \mathrm{E}+02$ & $1.72 \mathrm{E}-01$ & $-1.23 \mathrm{E}+00$ & $1.43 \mathrm{E}+02$ \\
\hline$E u$ & $1.15 \mathrm{E}+03$ & 4.79E-01 & $1.16 \mathrm{E}+01$ & $8.74 \mathrm{E}+02$ \\
\hline $\mathrm{Fe}$ & $7.15 \mathrm{E}-02$ & $1.49 \mathrm{E}-01$ & $3.52 \mathrm{E}-03$ & $1.48 \mathrm{E}-02$ \\
\hline$G a$ & $4.39 \mathrm{E}+02$ & $1.50 \mathrm{E}-01$ & $-1.88 \mathrm{E}+01$ & $6.08 \mathrm{E}+02$ \\
\hline$G d$ & $1.20 \mathrm{E}+02$ & $3.08 \mathrm{E}-01$ & $-4.38 \mathrm{E}+00$ & $1.41 \mathrm{E}+02$ \\
\hline Graphite & $1.50 \mathrm{E}+01$ & $3.51 \mathrm{E}-01$ & NA & NA \\
\hline Ground Mica & $1.17 \mathrm{E}-01$ & $3.04 \mathrm{E}-01$ & $-3.33 E-03$ & 1.47E-01 \\
\hline$H g$ & $1.81 \mathrm{E}+01$ & 2.97E-01 & $2.56 \mathrm{E}+00$ & $-4.92 \mathrm{E}+00$ \\
\hline Ho & $5.54 \mathrm{E}+02$ & $1.31 \mathrm{E}-01$ & $1.68 \mathrm{E}+01$ & $3.61 \mathrm{E}+02$ \\
\hline I & $1.82 \mathrm{E}+01$ & $2.15 \mathrm{E}-01$ & $9.75 \mathrm{E}-01$ & $9.39 E+00$ \\
\hline$K$ & $3.34 \mathrm{E}-01$ & $3.60 \mathrm{E}-01$ & $2.84 \mathrm{E}-02$ & $7.82 \mathrm{E}-02$ \\
\hline$L a$ & $2.65 \mathrm{E}+01$ & $6.79 \mathrm{E}-01$ & $-3.95 E-01$ & $2.61 E+01$ \\
\hline Leather & $1.85 \mathrm{E}+00$ & $3.51 \mathrm{E}-01$ & NA & NA \\
\hline$L i$ & $2.55 \mathrm{E}+01$ & $1.90 \mathrm{E}-01$ & $1.82 \mathrm{E}-01$ & 7.86E-01 \\
\hline$L u$ & $3.38 \mathrm{E}+03$ & $1.62 \mathrm{E}-01$ & $-1.50 E+02$ & $3.92 \mathrm{E}+03$ \\
\hline$M n$ & $3.68 \mathrm{E}-01$ & $4.98 \mathrm{E}-01$ & $1.50 \mathrm{E}-02$ & $2.33 \mathrm{E}-01$ \\
\hline Mo & $2.04 \mathrm{E}+01$ & $9.30 \mathrm{E}-01$ & $-2.03 E-01$ & $2.22 \mathrm{E}+01$ \\
\hline$N$ & $1.08 \mathrm{E}+01$ & $3.78 \mathrm{E}+00$ & $-2.59 \mathrm{E}+00$ & $3.42 \mathrm{E}+01$ \\
\hline Natural Rubber & $1.51 \mathrm{E}+00$ & $4.21 \mathrm{E}-01$ & $1.02 \mathrm{E}-01$ & $5.94 \mathrm{E}-01$ \\
\hline$N b$ & $1.34 \mathrm{E}+01$ & $7.95 \mathrm{E}-03$ & $-4.72 \mathrm{E}-01$ & $1.53 \mathrm{E}+01$ \\
\hline
\end{tabular}

Slope of Linear Intercept of Linear Fit

Price Volatility 


\begin{tabular}{|c|c|c|c|c|}
\hline Element & Average Price & $\begin{array}{l}\text { Price } \\
\text { Volatility }\end{array}$ & $\begin{array}{l}\text { Slope of Linear } \\
\text { Fit }\end{array}$ & $\begin{array}{l}\text { Intercept of } \\
\text { Linear Fit }\end{array}$ \\
\hline$N d$ & $5.44 \mathrm{E}+01$ & $8.12 \mathrm{E}-01$ & $3.44 \mathrm{E}+00$ & $1.53 \mathrm{E}+01$ \\
\hline $\mathrm{Ni}$ & $1.24 \mathrm{E}+01$ & $4.56 \mathrm{E}-01$ & $3.61 \mathrm{E}-01$ & $9.14 \mathrm{E}+00$ \\
\hline$P$ & $4.48 \mathrm{E}-02$ & $3.48 \mathrm{E}-01$ & $3.21 \mathrm{E}-03$ & $1.59 \mathrm{E}-02$ \\
\hline$P b$ & $1.41 \mathrm{E}+00$ & $2.20 \mathrm{E}-01$ & $6.41 \mathrm{E}-02$ & $8.30 \mathrm{E}-01$ \\
\hline$P d$ & $1.21 \mathrm{E}+04$ & $4.04 \mathrm{E}-01$ & $1.97 \mathrm{E}+02$ & $1.04 \mathrm{E}+04$ \\
\hline $\operatorname{Pr}$ & $6.25 \mathrm{E}+01$ & $5.20 \mathrm{E}-01$ & $3.69 \mathrm{E}+00$ & $1.99 \mathrm{E}+01$ \\
\hline$P t$ & $2.71 \mathrm{E}+04$ & $2.22 \mathrm{E}-01$ & $1.32 \mathrm{E}+03$ & $1.52 E+04$ \\
\hline Rare Earths & $8.10 E+00$ & $2.29 \mathrm{E}-01$ & $-7.20 \mathrm{E}-01$ & $1.17 \mathrm{E}+01$ \\
\hline$R e$ & $2.77 \mathrm{E}+03$ & 7.07E-01 & $1.41 \mathrm{E}+02$ & $1.50 \mathrm{E}+03$ \\
\hline$S$ & $4.91 \mathrm{E}-02$ & $8.96 \mathrm{E}-01$ & $3.88 \mathrm{E}-03$ & $1.42 \mathrm{E}-02$ \\
\hline$S b$ & $4.41 \mathrm{E}+00$ & $3.45 \mathrm{E}-01$ & $4.47 \mathrm{E}-01$ & $3.88 \mathrm{E}-01$ \\
\hline Sc & $4.64 \mathrm{E}+03$ & $3.15 \mathrm{E}-01$ & $-1.26 \mathrm{E}+02$ & $5.08 \mathrm{E}+03$ \\
\hline Se & $4.46 \mathrm{E}+01$ & $5.68 \mathrm{E}-01$ & $3.53 \mathrm{E}+00$ & $1.28 \mathrm{E}+01$ \\
\hline Sheet Mica & $1.34 \mathrm{E}+02$ & $2.05 \mathrm{E}-01$ & $4.78 \mathrm{E}+00$ & $3.45 E+01$ \\
\hline$S m$ & $2.00 \mathrm{E}+02$ & $4.56 \mathrm{E}-01$ & $-2.01 E+01$ & $3.51 \mathrm{E}+02$ \\
\hline$S n$ & $1.04 \mathrm{E}+01$ & $2.57 \mathrm{E}-01$ & $8.48 \mathrm{E}-01$ & $2.72 \mathrm{E}+00$ \\
\hline$T a$ & $1.15 \mathrm{E}+02$ & $6.38 \mathrm{E}-01$ & $1.68 \mathrm{E}+00$ & $9.98 \mathrm{E}+01$ \\
\hline$T b$ & $8.40 \mathrm{E}+02$ & $5.41 \mathrm{E}-01$ & $2.80 \mathrm{E}+01$ & $4.62 E+02$ \\
\hline$T e$ & $1.14 \mathrm{E}+02$ & $7.26 \mathrm{E}-01$ & $6.90 \mathrm{E}+00$ & $5.16 \mathrm{E}+01$ \\
\hline Th & $1.19 \mathrm{E}+02$ & $3.52 \mathrm{E}-01$ & $1.53 \mathrm{E}+01$ & $1.93 \mathrm{E}+01$ \\
\hline Ti & $9.54 \mathrm{E}-02$ & $4.31 \mathrm{E}-01$ & $3.40 \mathrm{E}-03$ & $6.48 \mathrm{E}-02$ \\
\hline $\mathrm{Tm}$ & $2.02 E+03$ & $1.67 \mathrm{E}-01$ & $-1.39 E+02$ & $2.92 E+03$ \\
\hline V & $1.04 \mathrm{E}+01$ & $6.92 \mathrm{E}-01$ & $2.53 \mathrm{E}-01$ & $8.08 \mathrm{E}+00$ \\
\hline$w$ & $1.66 \mathrm{E}+01$ & $3.03 \mathrm{E}-01$ & $1.40 \mathrm{E}+00$ & $4.08 \mathrm{E}+00$ \\
\hline$\gamma$ & $6.81 \mathrm{E}+01$ & $4.15 \mathrm{E}-01$ & $-3.91 E+00$ & $9.26 \mathrm{E}+01$ \\
\hline$Y b$ & $3.45 E+02$ & $1.28 \mathrm{E}-01$ & $4.87 \mathrm{E}+00$ & $2.59 E+02$ \\
\hline$Z n$ & $1.44 \mathrm{E}+00$ & $3.51 \mathrm{E}-01$ & $4.32 \mathrm{E}-02$ & $1.05 \mathrm{E}+00$ \\
\hline$Z r$ & $7.46 \mathrm{E}-01$ & $5.82 \mathrm{E}-01$ & $6.24 \mathrm{E}-02$ & $1.84 \mathrm{E}-01$ \\
\hline
\end{tabular}

Sheet and Ground Mica prices taken from USGS for the years 2006-2015 ${ }^{1}$. Natural Rubber Price data from Singapore Commodities Exchange ${ }^{2}$.

Prices for battery-grade graphite are harder to find. A report by an investment research firm, Edison ${ }^{3}$ puts the price of synthetic graphite between $\$ 10000$ per ton to $\$ 20000$ per ton. We use the average price of $\$ 15000$ per ton. Since we don't have price volatility data, we use the median price volatility of all materials. We use synthetic graphite price based on the observation by Olson et al that "Currently, primary synthetic graphite derived from petroleum coke is used in the anode of most lithium-ion batteries" 4

Leather prices are similarly difficult to find and vary widely based on quality, and measured by sqft rather than weight. We use the average global price of hides from 1998-2015 and use the median price volatility. ${ }^{5}$ 


\section{SI3. Performance Evaluation for Estimation of NoType Composition}

To better estimate composition of NoType parts, we make use of the two facts: 1 ) various suppliers produce equivalent parts and 2) each supplier has a unique policy for labeling compositional information as confidential. In light of this, we train a k-nearest neighbors (KNN) model to identify the parts most similar to the NoType part ${ }^{6}$. A distinct model was developed for each vehicle where the training dataset comprised all fully detailed parts (i.e., parts without data omissions) from all six of the other vehicles. Each part in the training data is described by a part number and its mass percent composition for 76 elements. For each NoType part, we predict the three nearest neighbors of all parts, based on a Euclidian distance of the elemental mass. We then estimate which elements have a higher average composition in the three nearest neighbors than the composition in the NoType part. We distribute the mass of the hidden material to these elements, based on a weighted average where the weights are determined by the difference in mass between the actual and predicted compositions.

To test this algorithm, we take $20 \%$ of the dataset (only taking parts with no missing information) and randomly delete an element from the part data. We then predict the part it was originally and test the algorithm in two ways. The stringent test classifies a prediction as successful only if the nearest neighbor of the modified part is the original part. For the relaxed test, a prediction is classified as successful when any of the three nearest neighbors is the original part. Results of this analysis as the number of omitted materials increases from one to five is shown in Figure SI.2. For both tests, our algorithm performs well. If only one element is omitted, success exceeds $90 \%$ (Relaxed 98\%, Stringent 95\%). Although the accuracy of prediction decreases as more materials are omitted, even when five materials are omitted, the algorithm can still correctly identify the actual part more than $80 \%$ of the time and it is one of the three most likely parts more than $90 \%$ of the time. These results provide confidence that this algorithm improves our estimate of vehicle composition.

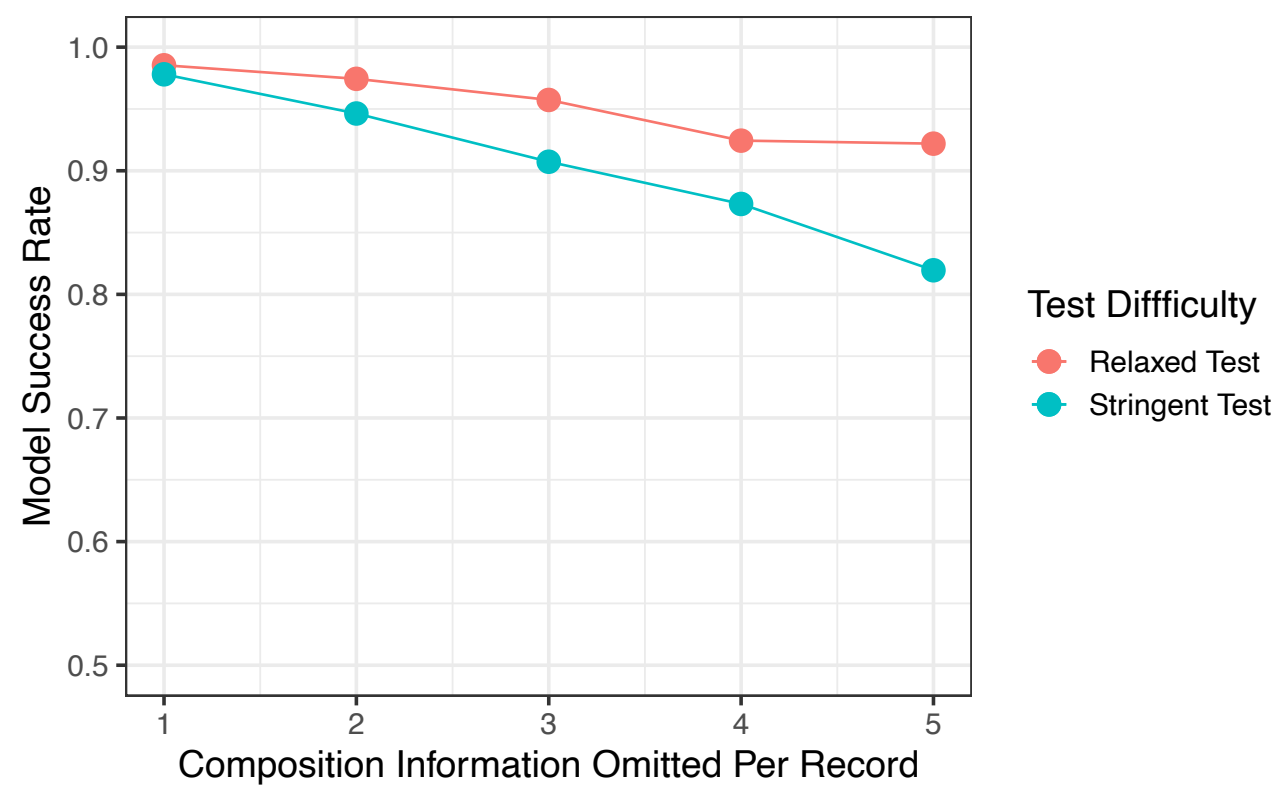

Figure SI 1. Performance of KNN model to estimate missing compositional information based on known composition. Performance as more compositional information is hidden (omitted from the test set). Stringent test is a success only if nearest neighbor is the original part. Relaxed test is a success if any of the three nearest neighbors is original part. 
SI4. Sensitivity Analysis of Exposure Calculation

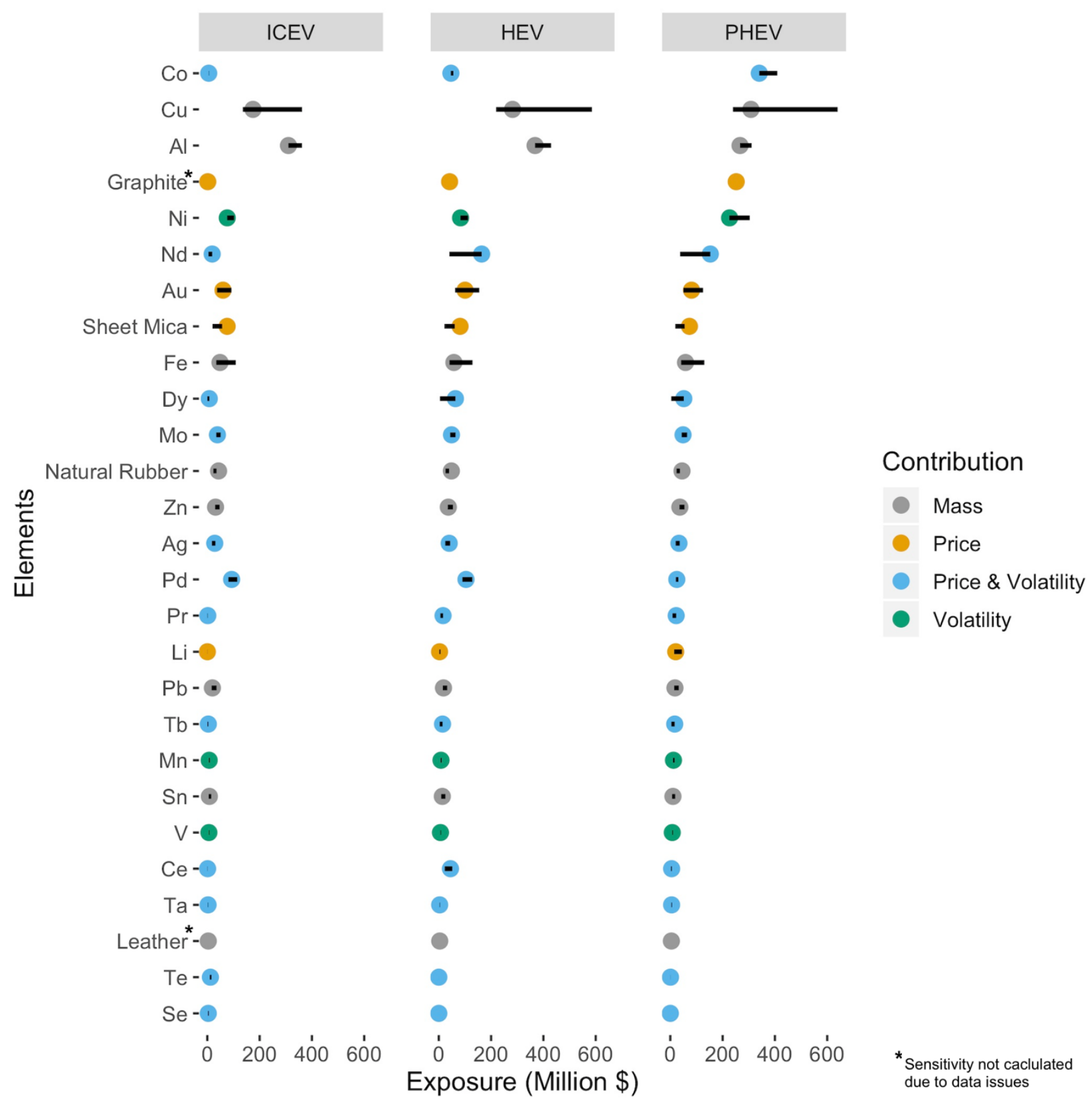

Figure SI 2: Variability in exposure contribution from each material in three vehicle fleets: a) all ICEV, b) all HEV and c) all PHEV. Each fleet has a ratio of 2 sedans to 1 SUV. Sensitivity calculated from the bootstrap metho" described in Methodology. 


\section{SI5. Comparison with material composition reported by Ortego et al for selected elements}

Table SI.5: Comparison of our estimates of material compositions (selected materials with high exposure scores) against those reported by Ortego et al ${ }^{7}$ who collected data from several sources, and previous work by some of the authors of this paper ${ }^{8}$. For comparison, we use the most similar vehicles (midsize ICEV and PHEV) although our vehicles are larger in size than those reported by Ortego et al.

\begin{tabular}{|c|c|c|c|c|c|}
\hline & $\begin{array}{r}\text { Ortego et al } \\
\text { ICEV }\end{array}$ & $\begin{array}{r}\text { Field et al }^{8} \\
\text { ICEV }\end{array}$ & $\begin{array}{r}\text { Sedan ICEV } \\
\text { (average of } \\
\text { Sedan } 1 \text { and } 2 \text { ) }\end{array}$ & $\begin{array}{r}\text { Ortego et al } \\
\text { PHEV (Li:ion) }\end{array}$ & $\begin{array}{r}\text { Sedan PHEV } \\
\text { (Li:ion) }\end{array}$ \\
\hline Al & $9.42404 \%$ & $9.12409 \%$ & $11.47194 \%$ & $10.78337 \%$ & $9.35871 \%$ \\
\hline Au & $0.00000 \%$ & $0.00015 \%$ & $0.00015 \%$ & $0.00002 \%$ & $0.00025 \%$ \\
\hline Co & $0.00000 \%$ & $0.00365 \%$ & $0.00312 \%$ & $0.20686 \%$ & $0.19090 \%$ \\
\hline $\mathrm{Cu}$ & $2.42967 \%$ & $2.26277 \%$ & $1.75152 \%$ & $4.51304 \%$ & $2.90626 \%$ \\
\hline Dy & $0.00125 \%$ & $0.00044 \%$ & $0.00026 \%$ & $0.01264 \%$ & $0.00055 \%$ \\
\hline $\mathrm{Fe}$ & $68.72498 \%$ & $58.39416 \%$ & $58.44674 \%$ & $61.49077 \%$ & $58.38743 \%$ \\
\hline $\mathrm{Li}$ & $0.00012 \%$ & Not Reported & $0.00050 \%$ & $0.17101 \%$ & $0.07231 \%$ \\
\hline $\mathrm{Nd}$ & $0.01381 \%$ & $0.00730 \%$ & $0.00613 \%$ & $0.04211 \%$ & $0.03307 \%$ \\
\hline $\mathrm{Ni}$ & $0.15175 \%$ & $0.16058 \%$ & $0.16627 \%$ & $1.22418 \%$ & $0.29747 \%$ \\
\hline $\mathbf{P d}$ & $0.00011 \%$ & Not Reported & $0.00024 \%$ & $0.00007 \%$ & $0.00012 \%$ \\
\hline Total $(\mathrm{kg})$ & $1,173,000$ & $1,370,000$ & $1,781,569$ & 1,311,000 & $1,848,721$ \\
\hline
\end{tabular}


SI6. Vehicle Mass and Exposure after filling in missing data, disaggregated by type of omission

Figures SI4 shows the mass and exposure per vehicle after filling in the different kinds of incomplete data. A large proportion of the incomplete data, by mass, is of 'ObscureType'. Given that this data had textual descriptions of materials we were able to get rid of this error in our exposure calculations. Most of the 'ObscureType' materials were organic and therefore did not contribute much to the exposure as seen in Figure SI2. The second largest error in data (by mass) is 'Unreported' data. The Sedans had much lesser Unreported data than the SUVs. This is likely because the Sedans we used in our analysis were 2019 model vehicles while the SUVs were 2020 model. This gave suppliers more time to report information for the sedans. The final type of data error that we corrected was the 'NoType' data. This is composition that suppliers report as being confidential- what is interesting is that while 'NoType' data is a low proportion of vehicle mass, it is a higher proportion of the vehicle exposure. This suggests that suppliers are deliberately hiding material that is more important from a vulnerability point of view and this could be a potential blind spot for manufacturers using IMDS data to understand their vulnerability.

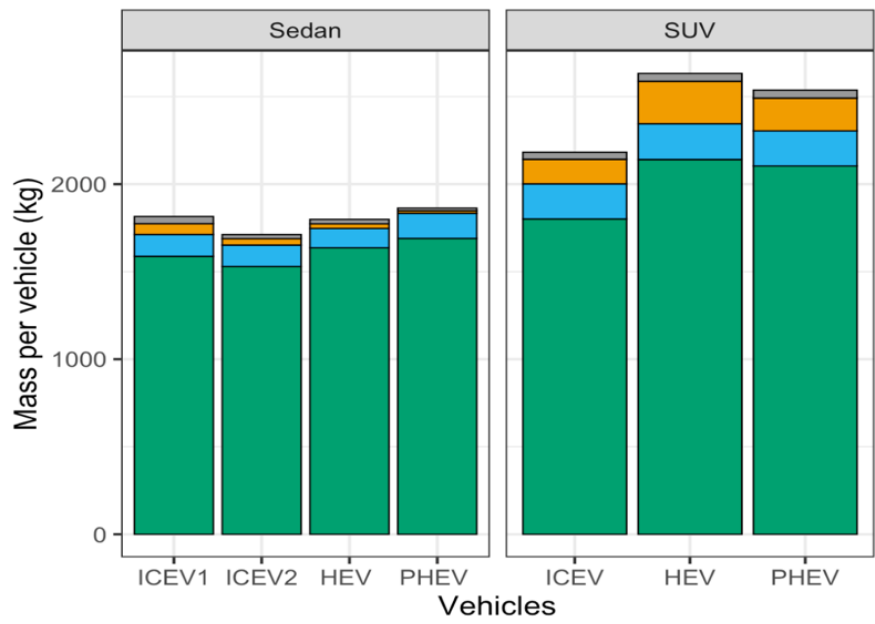

Data Omission Type

NoType

Unreported

ObscureType

OriginalData

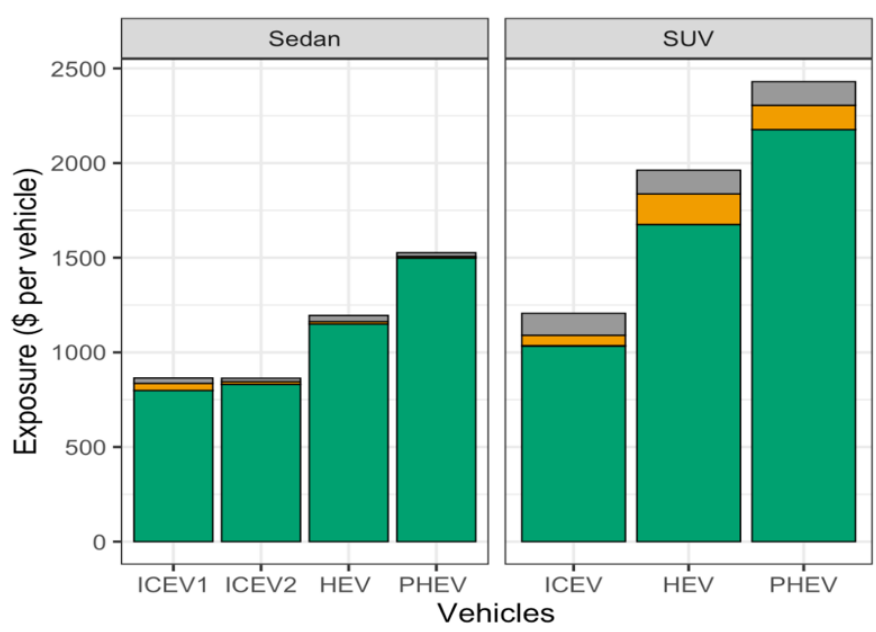

Data Omission Type

$\square$ NoType

Unreported

ObscureType

OriginalData

Figure SI 3: Mass and Exposure of each vehicle in our dataset (after applying filling in methods), disaggregated by type of mossing information: 


\section{Bibliography for Supporting Information}

(1) U.S. Geological Survey. Historical statistics for mineral and material commodities in the United States (2015 version): U.S. Geological Survey Data Series 140.

(2) World Bank. World Bank Commodity Price Data (The Pink Sheet): Monthly Prices.

(3) Hayes, T. Graphite for Batteries. Edison Rep. Met. Min. 2016.

(4) Olson, D. W.; Virta, R. L.; Mahdavi, M.; Sangine, E. S.; Fortier, S. M. Natural Graphite Demand and Supply $\backslash$ Implications for Electric Vehicle Battery Requirements. Spec. Pap. Geol. Soc. Am. 2016. https://doi.org/10.1130/2016.2520(08).

(5) International Monetary Fund. Global price of Hides [PHIDEUSDM] https://fred.stlouisfed.org/series/PHIDEUSDM (accessed Nov 23, 2020).

(6) Cover, T. M.; Hart, P. E. Nearest Neighbor Pattern Classification. IEEE Trans. Inf. Theory 1967, 13 (1), $21-27$. https://doi.org/10.1109/TIT.1967.1053964.

(7) Ortego, A.; Valero, A.; Valero, A.; Restrepo, E. Vehicles and Critical Raw Materials: A Sustainability Assessment Using Thermodynamic Rarity. J. Ind. Ecol. 2018, 22 (5), 1005-1015. https://doi.org/10.1111/jiec.12737.

(8) Field, F. R.; Wallington, T. J.; Everson, M.; Kirchain, R. E. Strategic Materials in the Automobile: A Comprehensive Assessment of Strategic and Minor Metals Use in Passenger Cars and Light Trucks. Environ. Sci. Technol. 2017. https://doi.org/10.1021/acs.est.6b06063. 